\title{
Faster growth rate in ad libitum-fed cats: a risk factor predicting the likelihood of becoming overweight during adulthood
}

\author{
Samuel Serisier ${ }^{1}$, Alexandre Feugier ${ }^{1}$, Claudie Venet ${ }^{1}$, Vincent Biourge ${ }^{1}$ and Alexander J. German ${ }^{2} *$ \\ ${ }^{1}$ Royal Canin Research Center, Aimargues, France \\ ${ }^{2}$ Department of Obesity and Endocrinology, Institute of Ageing and Chronic Disease, University of Liverpool, Liverpool, UK
}

(Received 10 September 2012 - Final revision received 8 January 2013 - Accepted 19 February 2013)

Journal of Nutritional Science (2013), vol. 2, e11, page 1 of 8

doi:10.1017/jns.2013.10

Abstract

In human subjects, the risk of becoming overweight $(\mathrm{OW})$ in adulthood is largely determined early in childhood. However, early-life factors have not been considered for feline obesity. A total of eighty colony cats, fed ad libitum, were studied; various breeds, ages and sex were included, with thirty-six (45\%) being OW and forty-four (55\%) being of ideal weight (IW). The effects of various factors (including age, sex, neuter status, breed (pure $v$. mixed), mean daily food intake (FI), housing status (indoor with outdoor access $v$. exclusively indoor) and body weight at 1 year of age $(\mathrm{BW} 1 \mathrm{y})$ ) on weight status were assessed. Initial statistical analyses identified BW1y as the main significant variable. Body weight (BW) and FI were then assessed between 1 and 8.5 years of age, with group differences (OW $v$. IW) noted for BW, which increased significantly with age only in the OW group $(P<0.001)$. However, no difference in BW $(P=0.17)$ was noted when BW1y was included as a covariate in the model. FI did not change with age in either group. Finally, given the importance of BW1y, changes in BW from 3 to 12 months were then assessed with BW at 3 months of age included as a covariate. Whereas at 3 months of age, no group difference in BW was observed, a faster rate of weight gain was seen in OW cats. In conclusion, as in human subjects, the rate of growth is a key risk factor for cats becoming OW, although the factors responsible are currently not known.

Key words: Obesity: Feline nutrition: Adolescents: Adiposity

Obesity is defined as the accumulation of excess body fat that adversely affects health ${ }^{(1)}$, and is one of the most important diseases affecting human subjects worldwide. Current estimates reveal that approximately $25 \%$ of men and women in the UK are obese $\left(\mathrm{BMI} \geq 30 \mathrm{~kg} / \mathrm{m}^{2}\right)$, and this suggests a threefold increase in prevalence since $1980^{(2)}$. Obese human subjects are at a greater risk of developing a number of diseases such as type 2 diabetes mellitus, hypertension, CHD, certain cancers (e.g. breast, ovarian and prostate), osteoarthritis, respiratory disease and reproductive disorders ${ }^{(1)}$. A number of longitudinal studies have identified adolescent obesity as a major risk factor for obesity in adulthood ${ }^{(3-9)}$. The risk factors for childhood and adolescent overweight (OW) are less clear, with various factors implicated, for example sex, birth weight, parent weight status, physical activity in childhood, television viewing in childhood, socio-economic status and education level ${ }^{(4-6,8,10-12)}$. Both heavy birth weight and rapid growth during infancy have also been identified as risk factors for obesity in adulthood ${ }^{(4,8,13)}$, but results are inconsistent regarding nutritional factors and dietary intake ${ }^{(8)}$, in part related to the fact that accurate data are difficult to obtain in longitudinal studies.

Problems with excess body weight (BW) are also a growing concern in many domesticated animal species including cats, and are known to lead to a range of medical disorders ${ }^{(14-16)}$. Recent studies have suggested that $20-52 \%$ of cats in the Westernised world are OW or obese ${ }^{(15,17-19)}$. Several cross-

Abbreviations: BCS, body condition score; BW, body weight; BW1y, body weight at 1 year of age; FI, food intake; IW, ideal weight; OW, overweight; VIP, variable importance in projection.

* Corresponding author: A. J. German, fax +44 151795 6101, email ajgerman@liv.ac.uk 
sectional studies have assessed the factors predisposing cats to becoming OW, and the factors associated with OW status include: neuter status $^{(15,18,20-23)}$, middle age ${ }^{(15,17,20,21,23)}$, underestimation of the body condition score (BCS) by the owner ${ }^{(17,24)}$, not living with dogs in the household ${ }^{(24)}$, mixed breed rather than pure breed $^{(21,22)}$, multi-cat households ${ }^{(22)}$ and longer day length ${ }^{(24)}$. However, dietary factors (including feeding frequency, feeding of premium foods (i.e. superior palatability, digestibility, and ingredient quality) and the use of treats) are less consistent with some $^{(15,18,22-24)}$, but not all ${ }^{(19)}$, studies suggesting associations. Unfortunately, evidence from longitudinal studies is lacking regarding risk factors for excess weight in cats and the influence of early life factors. Since cats are an outbred species, live in the same environment as human subjects, and share some of the same disease risks, comparative information on cats can also be of interest for understanding human obesity. Further, studies are arguably easier to conduct since lifespan is shorter.

The aims of the current study were, firstly, to identify risk factors for the development of excess weight in cats in late adulthood, by assessing a variety of signalment factors and early life factors. The work described is an observational study, in a colony of cats owned by a commercial pet food company, designed to assess the effects of various factors on food intake (FI) and the likelihood of cats fed dry diets ad libitum becoming OW.

\section{Experimental methods}

\section{Animals}

A total of eighty cats were included in the study, representing fourteen different breeds, with thirty-six males (thirty-four neutered) and forty-four females (twenty-four neutered). Median age, at the study end, was $7 \cdot 6$ years (range $1.5-10 \cdot 6$ years). Of these cats, thirty-six were determined to be OW (OW group; BCS $>5 / 9$ ), while the remaining forty-four were in ideal body condition (ideal weight (IW) group; BCS $=5 / 9$ ). In all cats, BCS was ideal (e.g. 5/9) ${ }^{(25)}$ at 12 months of age.

Of these eighty cats, a subset of forty-two cats was identified where continuous BW data were available from 1 to 8.5 years, and FI data from 3.5 to 8.5 years. The distribution of all variables, for this subgroup, was approximately the same as for the eighty cats (Table 1) except for the breed whereby more pedigree cats were present in the forty-two-cat group subset than in the population as a whole $(P=0 \cdot 036)$. Of the forty-two-cat subgroup, monthly BW data were also available from 3 months of age in sixteen of the cats. Again, the distribution of variables was broadly similar to the population of eighty cats (Table 1).

\section{Housing and husbandry}

All cats were maintained in the colony of a commercial pet food company, and were not client-owned. Housing and treatment protocols adhered to European regulatory rules for animal welfare; all experimental protocols complied with the European Union guidelines on animal welfare and were approved by the Royal Canin committee for animal ethics and welfare. Cats were housed in closed indoor-outdoor runs; sixty-two of the cats had unlimited outdoor access, while the remaining eighteen were housed exclusively indoors (Table 1). The size of all runs was $27 \mathrm{~m}^{2}$, and there were a maximum of eight cats per run, and the cat groups remained the same throughout the study. The runs with outdoor access

Table 1. Summary data for the eighty cats and their comparison with the forty-two- and sixteen-cat subgroups (Number and percentage; median values and ranges for age, food intake and body weight at 1 year of age)

\begin{tabular}{|c|c|c|c|c|c|c|c|}
\hline \multirow[b]{2}{*}{ Factors } & \multicolumn{2}{|c|}{ Eighty cats } & \multicolumn{2}{|c|}{ Forty-two cats* } & \multicolumn{2}{|c|}{ Sixteen cats $\dagger$} & \multirow[b]{2}{*}{$P$} \\
\hline & $n$ & $\%$ & $n$ & $\%$ & $n$ & $\%$ & \\
\hline \multicolumn{8}{|l|}{ BCS } \\
\hline Ideal $=5 / 9$ & 44 & 55 & 25 & 59 & 8 & 50 & \multirow[t]{2}{*}{0.788} \\
\hline$O W>5 / 9$ & 36 & 45 & 17 & 41 & 8 & 50 & \\
\hline \multicolumn{8}{|l|}{ Neuter status } \\
\hline Intact & 22 & 28 & 12 & 29 & 4 & 25 & \multirow[t]{2}{*}{0.964} \\
\hline Neutered & 58 & 72 & 30 & 71 & 12 & 75 & \\
\hline \multicolumn{8}{|l|}{ Sex } \\
\hline Male & 36 & 45 & 18 & 43 & 7 & 44 & \multirow[t]{2}{*}{0.974} \\
\hline Female & 44 & 55 & 24 & 57 & 9 & 56 & \\
\hline \multicolumn{8}{|l|}{ Pedigree status } \\
\hline Pedigree & 55 & 69 & 35 & 83 & 10 & 62 & \multirow[t]{2}{*}{0.036} \\
\hline Mixed breed & 35 & 31 & 7 & 17 & 6 & 38 & \\
\hline \multicolumn{8}{|l|}{ Housing } \\
\hline Exclusively indoor & 18 & 23 & 13 & 31 & 3 & 19 & \multirow[t]{2}{*}{0.497} \\
\hline \multirow[t]{2}{*}{ Outdoor access } & 62 & 77 & 29 & 69 & 13 & 81 & \\
\hline & Median & Range & Median & Range & Median & Range & \\
\hline Age at study end (years) & 7.6 & $1.5-10 \cdot 6$ & 8.5 & & 8.5 & & - \\
\hline Food intake $(\mathrm{g} / \mathrm{d})$ & 53 & 33-97 & 52 & $33-97$ & 53 & $39-97$ & - \\
\hline Food intake (g/kg per d) & 14 & $8-21$ & 14 & 8-18 & 13 & $8-18$ & - \\
\hline Body weight at 1 year $(\mathrm{kg})$ & 3.41 & $1 \cdot 76-6 \cdot 50$ & $3 \cdot 31$ & $1 \cdot 81-6.50$ & 3.77 & $1 \cdot 81-6 \cdot 50$ & - \\
\hline
\end{tabular}

BCS, body condition score; OW, overweight.

* Body weight data available from 12 months and food intake data available from 3.5 years to the end of the study.

† Body weight data available from 3 months to the end of the study. The $P$-values quoted are for a comparison of the proportions of cats in each population, for each variable listed, made using a Fisher's exact test. 
Table 2. Dietary composition of the basal diet used for the study cats

\begin{tabular}{lccc}
\hline Criterion & Diet composition & & \\
\hline ME content & $16161 \mathrm{~kJ} / \mathrm{kg}(3860 \mathrm{kcal} / \mathrm{kg})$ & \\
& Per $100 \mathrm{~g}$ & $\mathrm{~g} / 1000 \mathrm{kcal}$ & $\mathrm{g} / \mathrm{MJ}$ \\
Moisture & $\mathrm{DM}$ & $(\mathrm{ME})$ & $(\mathrm{ME})$ \\
Crude protein & 7 & 18 & 4 \\
Crude fat & 32 & 83 & 20 \\
Crude fibre & 15 & 39 & 9 \\
Total dietary fibre & 5.5 & 14 & 3 \\
Ash & 11 & 28 & 7 \\
Nitrogen-free extract & 6.8 & 18 & 4 \\
& 33.7 & 87 & 21 \\
\hline
\end{tabular}

ME, metabolisable energy measured in animal trials according to the 2010 American Association of Feed Control Officials (AAFCO) protocols ${ }^{(26)}$.

were divided into an indoor part (of $13 \mathrm{~m}^{2}$ ) and an outdoor part (of $14 \mathrm{~m}^{2}$ ). Inside, the temperature varied between 18 and $24^{\circ} \mathrm{C}$, depending on season, and artificial light was provided between 07.30 and 17.00 hours if natural light was judged to be insufficient by animal handlers. For all cats, caregivers stimulated play behaviour for approximately $2 \mathrm{~h}$ per run per day. All cats remained healthy for the duration of the studies.

This population of eighty cats was used in feeding performance studies during the period of the study (June 2001 to December 2009). For $85 \%$ of the time, the whole group of cats was fed the same basal diet ad libitum (Table 2). For the remaining $15 \%$ of the time, the whole group was offered two dry expanded balanced diets ad libitum. Although the group was always fed the same regimen, the diets themselves would be changed on a daily basis and each cat had access to its own food station by microchip recognition. Despite the use of a range of diets, there was little variation in composition and metabolisable energy content over time (Table 3). From December 2004 onwards, individual FI was recorded daily using electronic weigh scales (M-Tronic Paris; accurate to within $0.5 \mathrm{~g}$ ), and the mean FI (in g) was then automatically calculated. BW was also recorded, on a monthly basis, using the same calibrated electronic weigh scale (SG16000; Mettler Toledo; accurate to within $1 \mathrm{~g}$ ). In addition, BCS was also scored monthly, although only the data for 1 year of age and the end of the study are described here. The BCS assignments were made group-consensus: the same six individuals scored the cats independently and then compared scores.
When there was a discrepancy, the lead author (S. S.) made the final decision.

\section{Statistical analysis}

All data were analysed using the Statistical Analysis Systems Institute package (SAS version 8; SAS Institute Inc.), and Tanagra version 1.4.38 (Tanagra project; http://eric. univ-lyon2.fr/ $\sim$ ricco/tanagra/en/tanagra.html). Significance was set at $5 \%(P<0.05)$ for two-sided analyses. Exact $P$ values are reported throughout, except for the partial least squares discriminant analysis (mentioned later), because the software (Tanagra) did not display them. Except where indicated, results are expressed either as median (range) or as least-square means (SEM).

Data from the entire population of eighty cats were first used to identify risk factors associated with being OW (i.e. being in the OW group). The factors tested included age (young adult, $<7.6$ years (median population age) $v$. mature adult, $>7.6$ years), sex, neuter status, pedigree status (pedigree $v$. mixed), housing status (indoor with outdoor access $v$. exclusively indoor), and body weight at 1 year of age (BW1y; BCS was $5 / 9$ in all cats at this age). The mean daily FI (in $\mathrm{g} / \mathrm{d}$, and $\mathrm{g} / \mathrm{kg}$ current weight/d) was also assessed, in all cats, over a 12 -month period in 2009 (i.e. the last year of the study). Given that both binary and continuous variables were included, partial least squares discriminant analysis was performed. The Hosmer Lemeshow test was used to judge whether data fitted the statistical model well (i.e. goodness-of-fit). Results are expressed as variable importance in projection (VIP), with values greater than 1 considered to be important (at a significance level of $P<0 \cdot 05)^{(27)}$.

Next, data from the forty-two-cat subgroup were used to compare changes in BW and FI between cats that were OW or cats that were in ideal body condition at 8.5 years of age. $\mathrm{BW}$ data were assessed from 1 to 8.5 years of age, while FI data were assessed between 3.5 and 8.5 years of age. Tests performed included one-way ANOVA and the mixed procedure of SAS. For the latter analyses, group (OW or IW) and age were included as fixed effects, while cat was defined as a random term taking into account that several measurements were performed on the same cat. In some models, either the BW1y,

Table 3. Dietary composition of the $15 \%$ remaining diets used in palatability trials in the study cats (Median values and ranges)

\begin{tabular}{|c|c|c|c|c|c|c|}
\hline \multirow[b]{2}{*}{ Criterion } & \multicolumn{6}{|c|}{ Diet composition } \\
\hline & Median & Range & Median & Range & Median & Range \\
\hline ME content $(\mathrm{kJ} / \mathrm{kg})$ & 16281 & $12916-18597$ & & & & \\
\hline \multirow[t]{2}{*}{ ME content (kcal/kg) } & 3895 & 3090-4449 & \multirow{2}{*}{\multicolumn{2}{|c|}{ g/1000 kcal (ME) }} & & \\
\hline & per $100 \mathrm{~g} \mathrm{DM}$ & & & & $\mathrm{g} / \mathrm{MJ}(\mathrm{ME})$ & \\
\hline Moisture & 5.5 & $5 \cdot 5-8$ & 14 & $12-23$ & 3 & $3-5$ \\
\hline Crude protein & 34 & $23-46$ & 84 & $61-127$ & 20 & $15-30$ \\
\hline Crude fat & 15 & $9-25$ & 39 & $28-56$ & 9 & $7-13$ \\
\hline Crude fibre & 4.3 & $1 \cdot 3-14 \cdot 1$ & 11 & $3-46$ & 3 & $1-11$ \\
\hline Total dietary fibre & 11 & $6 \cdot 1-23$ & 28 & $14-74$ & 7 & $3-18$ \\
\hline Ash & 7.4 & $5 \cdot 2-9 \cdot 4$ & 19 & $13-27$ & 5 & $3-7$ \\
\hline Nitrogen-free extract & 33.4 & $24 \cdot 8-44.4$ & 88 & $58-118$ & 21 & $14-28$ \\
\hline
\end{tabular}

ME, metabolisable energy measured in animal trials according to the 2010 American Association of Feed Control Officials (AAFCO) protocols ${ }^{(26)}$. 
Table 4. Risk factors for overweight in a population of eighty colony cats*

\begin{tabular}{lc}
\hline Factors & VIP \\
\hline Age (young $v$. mature) & 0.76 \\
Sexual status (intact $v$. neutered) & 1.01 \\
Sex (male $v$. female) & 0.89 \\
Pedigree status (pedigree $v$. mixed breed) & 0.95 \\
Housing (exclusively indoor $v$. outdoor access) & 0.46 \\
Food intake (g/kg per d) & 0.76 \\
Body weight at 1 year $(\mathrm{kg})$ & 1.71 \\
\hline
\end{tabular}

* Partial least squares discriminant analysis was performed to assess the effect of binary and continuous variables on the risk of being overweight. The Hosmer Lemeshow test was used to judge the goodness of fit, and results are expressed as variable importance in projection (VIP), with values greater than 1 considered to be important (at a significance level of $P<0.05$ ). Food intake data are expressed as $\mathrm{g}$ of food per $\mathrm{kg}$ of the current body weight during the 12-month measurement period in 2009 .

or the BW corresponding to each FI measurement, was included as a covariate in the analysis.

Finally, the sixteen-cat subgroup was used to examine the change in weight during the growth period between cats that were OW or cats that were in ideal body condition at 8.5 years. Tests performed again included one-way ANOVA and the mixed procedure of SAS (SAS Institute, Inc.). In this analysis, BW at 3 months of age was included as a covariate.

\section{Results}

\section{Assessment of risk factors for being overweight in eighty colony cats fed ad libitum}

Risk factors for being OW were examined, in a cross-sectional manner, in a population of eighty cats (Table 4): BW1y (OW group: median $3.95 \mathrm{~kg}(2.22-6.50 \mathrm{~kg})$; IW group: median 3.02 $\mathrm{kg}(1.76-5.46 \mathrm{~kg})$; VIP $1.71, P<0.05)$ was the main factor found to be of significance; the only other effect noted was sexual status, but this was at the limit of significance (intact $v$. neutered, VIP 1.01, $P<0 \cdot 05)$. At this stage, median BCS was 7 (6-9) and 5 (5-5) in OW and IW groups, respectively. As a result, changes in BW and FI, and their effect on subsequent weight status, were examined in a subgroup of forty-two cats with available data.
Longitudinal study of risk factors for becoming overweight, from 1 to 8.5 years of age, in forty-two colony cats

When changes in BW were assessed, between 1 and 8.5 years of age, both age and group effects were noted. With regard to age, BW increased steadily as cats got older $(P<0 \cdot 001$; Fig. 1). With regard to group, BW was greater in the OW group $(P<$ 0.001 ; Fig. 1) such that at 8.5 years of age, the BW of those in the OW group (median $4.88 \mathrm{~kg}(3.56-10.10 \mathrm{~kg})$ ) was greater than those in the IW group (median $3.50 \mathrm{~kg}(2.34-4.59 \mathrm{~kg}) ; P$ $<0 \cdot 001)$. At this stage, the median BCS in the OW group was 7 (6-9). The group difference in BW was present at each timepoint, and was also present at 1 year of age when the BCS of all cats was ideal (5/9). Further analysis also revealed a groupage interaction, with $\mathrm{BW}$ increasing progressively more in the OW group than in the IW group $(P<0 \cdot 001)$. In this respect, the median difference in BW between 2.5 and 8.5 years of age was $22 \%$ (range $2-62 \%$ ) in OW cats and 2\% (range -17 to $24 \%$ in IW cats.

Given that the differences in BW were already present at 1 year of age, the analysis was repeated with BW1y included as a covariate. With this analysis, the overall group effect disappeared $(P=$ $0 \cdot 17)$, but the group-age interaction remained $(P<0 \cdot 001)$ : again there was no significant change in weight of cats in the IW group, while the weight of cats in the OW group progressively increased, becoming significantly different from the BW at 1 year of age from 4 years of age onwards $(P<0 \cdot 05)$.

Next, FI was assessed in the forty-two cats, for a period of at least 5 years, between 3.5 and 8.5 years of age. As with BW, FI at 8.5 years of age in the OW group was greater than that in the IW group (OW $v$. IW: $60 \cdot 7$ (SEM 2.6) g/d $v .50 \cdot 3$ (SEM 2.2) g/d; $P<0 \cdot 001)$. Although the group effect was evident at each time point $(P=0.002)$, no age-group interaction was evident for FI $(P=0 \cdot 75)$. Further, when BW1y was included as a covariate in the model, the overall group effect disappeared (OW $v$. IW: 56.1 (SEM 2.2) g/d $v$. 53.4 (SEM 1.8) g/d; $P=0.37$ ). The group effect also disappeared when the BW corresponding to each FI measurement was included as a covariate in the analysis (OW $v$. IW: 55.9 (SEM 2.2) g/d $v .53 .4$ (SEM 1.8) g/d; $P=0.41$ ).

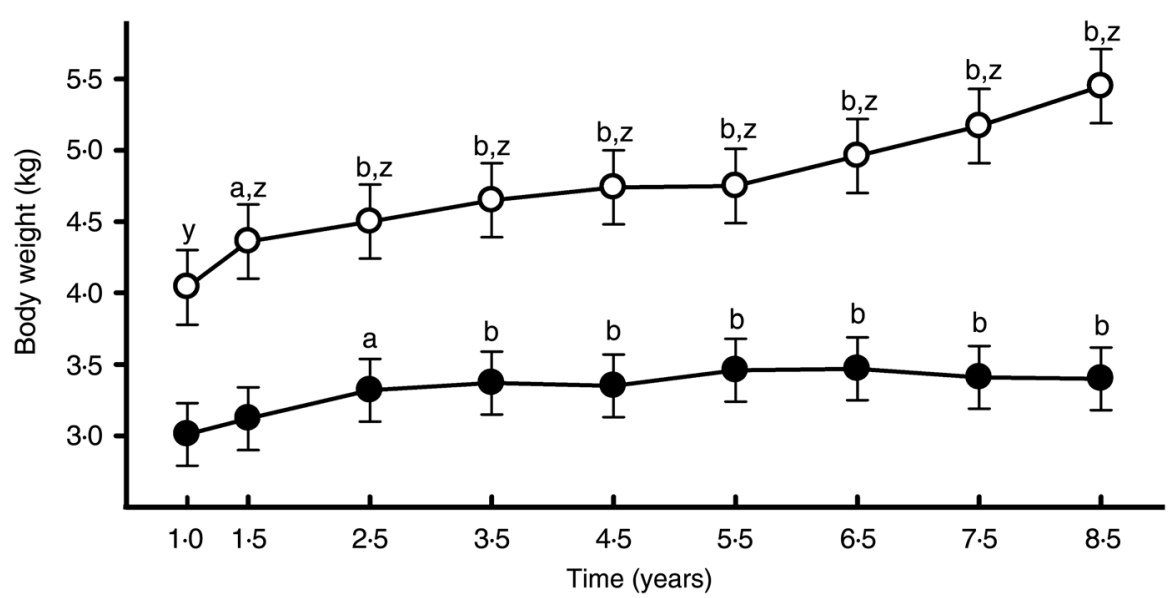

Fig. 1. Body weight assessed, in forty-two colony cats between 1 and 8.5 years of age. Values represent least squares means, while error bars represent sEM. The open and filled symbols represent data for cats that were overweight and of ideal weight at 8.5 years of age, respectively. The letters identify significant differences compared with body weight at 1 year of age in the same group (a: $P<0.01$; b: $P<0.001)$ or differences between cat groups $(\mathrm{z}$ : $P<0.001)$. A groupage interaction $(P<0.001)$ was also identified, whereby body weight increased progressively more in the overweight group than in the ideal weight group. 


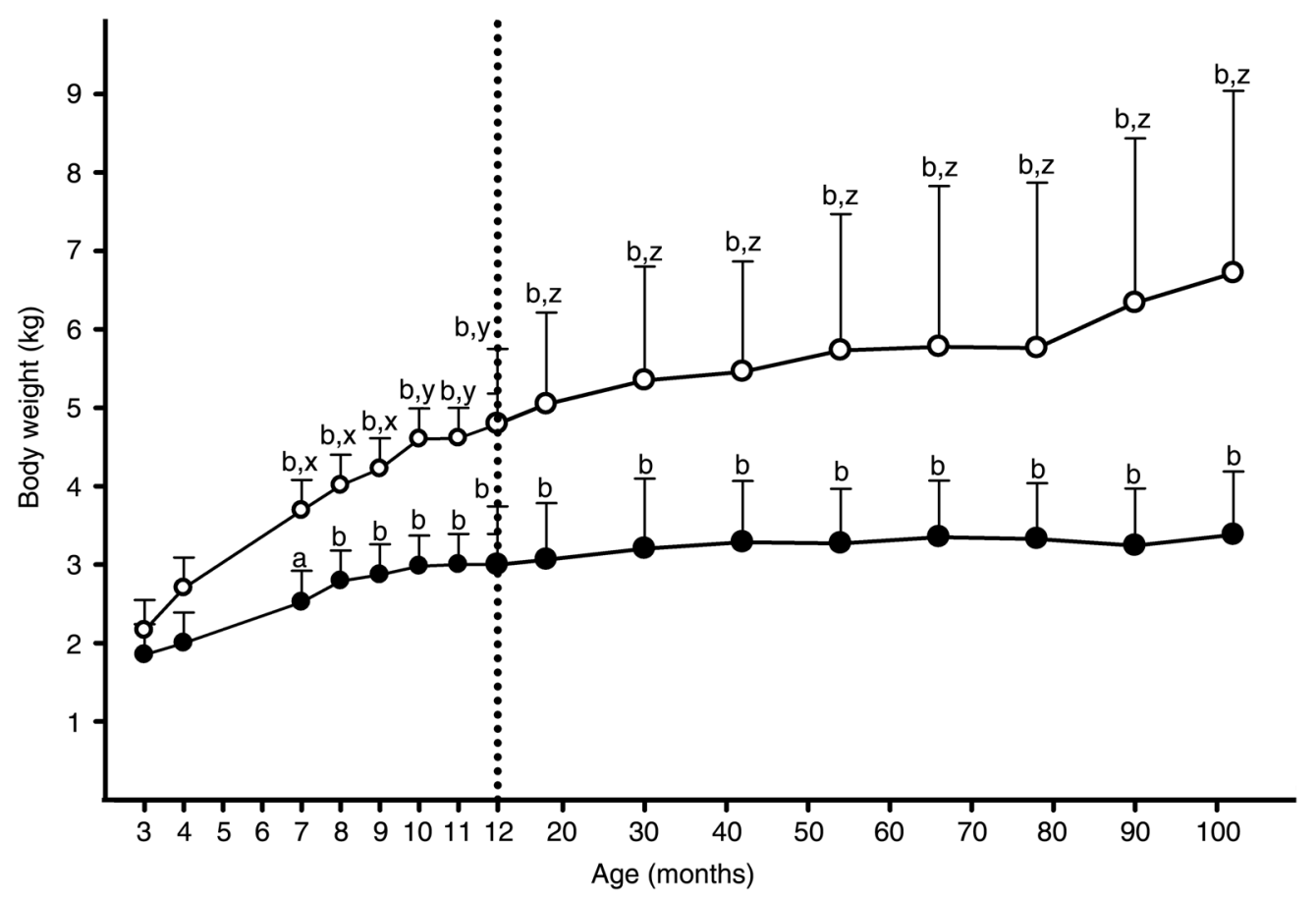

Fig. 2. Body weight assessed in sixteen colony cats monitored from 3 months to 8.5 years of age. Values represent least squares means, while error bars represent SD. The open and filled symbols represent data for cats that were overweight and of ideal weight at 8.5 years of age, respectively. The letters identify significant differences compared with body weight at 3 months of age in the same group (a: $P<0.05$; b: $P<0.001)$ or differences between cat groups (x: $P<0.05$; y: $P<$ $0.01 ; z: P<0.001)$. Both age (body weight significantly greater in older cats) and group (body weight significantly greater in overweight cats) effects were identified.

\section{Longitudinal study of risk factors for becoming overweight, from 3 months to 8.5 years of age, in sixteen colony cats}

Given that differences in both BW and FI during adult life were related to $\mathrm{BW} 1 \mathrm{y}$, we were interested in identifying whether such associations were evident at an even younger age. Therefore, a subgroup of sixteen cats (eight OW and eight IW) was selected, where BW had been recorded from 3 months of age, thus enabling weight change during growth to be studied. In this subset, the same effect on BW was evident as for the forty-two-cat subset: at 8.5 years of age, the BW of cats in the OW group was greater than that of cats in the IW group (OW v. IW: 6.61 (SEM 0.39) $\mathrm{kg} v .3 .38$ (SEM 0.39) kg; $P<0.001$; Fig. 2), but this group effect disappeared when BW1y was included as a covariate in the model $(P=0 \cdot 80)$. As before, a group-age interaction was evident in the initial model, with weight increasing progressively in the OW group but not in the IW group $(P<0.001$; Fig. 2$)$, and this effect remained when $\mathrm{BW} 1 \mathrm{y}$ was included as a covariate $(P<0 \cdot 001)$.

At 3 months of age, no difference in BW was observed between the two groups $(P=1 \cdot 0)$. When $\mathrm{BW}$ at 3 months of age was included as a covariate in a model of $\mathrm{BW}$ change from 3 to 12 months of age, it remained as a significant effect $(P=0.003)$. However, both a significant group effect $(P=$ 0.002) and age-group interaction were evident, with $\mathrm{BW}$ increasing more in cats of the OW group than in cats in the IW group $(P<0 \cdot 001)$. This was confirmed by the finding that the percentage change in weight from 3 to 12 months was greater in the OW group (median $117.2 \%$ (range 67.6$182.6 \%)$ ) compared with the IW group (median 59.6\% (range 18.7-120.3\%); $P=0.008$ ).
In light of these findings, partial least squares discriminant analysis was then used to determine the factors associated with being $\mathrm{OW}$ at 8.5 years, this time including percentage change in weight between 3 and 12 months, instead of BW1y. The factors that had a significant effect in the model were sex (VIP 1.56; $P<0.05$ ), and $\mathrm{BW}$ change between 3 and 12 months of age (VIP 1.12; $P<0 \cdot 05$; Table 5).

\section{Discussion}

The work reported here is the first longitudinal study to assess risk factors for becoming OW in domesticated cats. Only a small population was studied, but the fact that the population was followed for almost 8 years is a real strength. Although not

Table 5. Risk factors for overweight at 8.5 years in sixteen cats followed from 3 months of age*

\begin{tabular}{lc} 
Factors & VIP \\
\hline Sexual status (intact $v$. neutered) & 0.63 \\
Sex (male $v$. female) & 1.56 \\
Pedigree status (pedigree $v$. mixed breed) & 0.99 \\
Housing (exclusively indoor $v$. outdoor access) & 0.41 \\
Food intake (g/kg per d) & 0.88 \\
Body weight change from 3 to 12 months $(\%)$ & 1.12
\end{tabular}

VIP, variable importance in projection.

* Partial least squares discriminant analysis was performed to assess the effect of binary and continuous variables on the risk of being overweight. The Hosmer Lemeshow test was used to judge goodness of fit, and results are expressed as VIP, with values greater than 1 considered to be important (at a significance level of $P<0.05$ ). Food intake data are expressed as $\mathrm{g}$ of food per $\mathrm{kg}$ of the current body weight during the 12-month measurement period in 2009. Age was not included as a factor in the above analysis because all cats were approximately the same age. 
the complete lifespan, this period is sufficient to identify the majority of OW cats, in that most cats that are OW have become OW by middle age, and prevalence of obesity is less in older cats ${ }^{(15)}$. The key finding was that, within the population studied, two phenotypes of cats were identified: cats with the 'IW' phenotype regulated their FI and BW throughout life despite ad libitum access to food; cats with the 'OW' phenotype were already heavier at 12 months of age, and continued to gain weight progressively thereafter. Although many factors are undoubtedly involved, BW at 12 months of age was a key factor independent of breed, sex and neuter status. In a subset of the cats, we were able to examine weight changes from weaning, and this revealed that the rate of growth (as measured by percentage change in BW between 3 and 12 months of age) was a more important risk factor than BW1y. These findings mirror those of human studies, where rapid growth in infancy is a major risk factor for obesity in adulthood $^{(13,28,29)}$. Of course, the factors associated with this difference in growth rate are not clear, and warrant further study. These could include genetic and epigenetic differences, in utero factors, difference in physical activity levels, differences in feeding-related behaviour, digestibility differences and possible differences in gastrointestinal microbiota.

Although the development of the OW phenotype is probably multifactorial, genetic background is likely to be of critical importance. This is clearly the case in human subjects, with family, twin and adoption studies all suggesting the trait to be highly heritable, accounting for between 40 and $70 \%$ of the variation in $\mathrm{BMI}^{(30,31)}$. In a recent study of the ' $\mathrm{OW}$ ' phenotype in a cat colony from Switzerland, complex segregation analysis also revealed the likelihood of a strong genetic component in the development of OW, with a major gene model explaining segregation the best ${ }^{(32)}$. The population examined in that study was different from that of the current study, in that all cats were sexually intact, mixed breed and fed as a group rather than individually. In contrast, the current population of cats was relatively more diverse in terms of signalment (e.g. range of breeds, neutered and intact animals) and environmental factors (indoor-outdoor $v$. exclusively indoor) and, therefore, it is not clear whether similar findings would be evident. As a result, prospective genomic studies are needed, both on the current population and the wider pet cat community, to determine what genetic factors might be responsible for the differences identified, and to examine similarities with the OW phenotype identified in the Haring et al. study ${ }^{(32)}$.

In utero factors, probably acting through epigenetic mechanisms, may also be important for the early life differences between OW and IW cats. In this respect, there is emerging evidence in human subjects that the in utero environment may influence the likelihood of subsequent obesity in the offspring ${ }^{(33)}$. Factors implicated include excessive maternal weight gain during gestation ${ }^{(34)}$, low carbohydrate intake during pregnancy ${ }^{(35)}$, gestational diabetes mellitus ${ }^{(36)}$ and smoking during pregnancy ${ }^{(37)}$. Given that the domesticated cat is a pluriparous species, litter size might also be important. The influence of metagenomics has also been studied recently, with emerging work on possible maternal influences on metabolic and growth parameters in cats ${ }^{(38)}$. Further work on the effect of in utero and maternal factors on the growth of kittens and predisposition to OW is warranted.

Given that long-term success with weight management is disappointing ${ }^{(39)}$, more time should arguably be devoted to obesity prevention $^{(40)}$ and early identification of the at-risk population, so that efforts at prevention can be best focused. Although previous work on OW colony cats suggested that obese and lean phenotype cats could be identified by BCS as early as 8 months ${ }^{(38)}$, BCS of all cats was ideal (5/9) in the cats of the present study. This implies that BCS changes may not be sensitive enough to identify those at risk of obesity early in life. This is perhaps not surprising since current BCS systems have only been validated in adult cats, and a 1-unit change in BCS, on a nine-integer unit scale, requires a $\mathrm{BW}$ change of approximately $10-15 \%{ }^{(15,16)}$. However, the fact that the difference in median 12-month $\mathrm{BW}$ between two groups was greater than this $(22 \%)$ suggests that this is not only due to increased adiposity but also due to increased stature. This is similar to the situation in human beings, whereby rapid weight gain in infancy not only leads to greater adiposity but also taller height when skeletally mature ${ }^{(41)}$.

Based upon the findings of the present study, an alternative option would be to consider BW measurement at 12 months, since this was found to be a key marker of risk of OW later in life. Such an approach would have the benefit of ease of application, since a single measurement at 12 months, using precise and accurate weighing scales, is all that would be required. However, the main problem would be that BW is also likely to be affected by sex, breed, size of body frame, and the geographical population (i.e. country and continental differences), so that an appropriate cut-off would need to be established for different cat populations. An alternative approach would be to use percentage change in $\mathrm{BW}$ between 3 and 12 months. Although requiring two BW measurements (which would ideally need to be performed on the same weighing scales), such a measure would correct for sex, breed differences and geographical differences in populations and, therefore, be more widely applicable. Further, the use of measurements at 3 months and 12 months could readily be fitted into the normal pattern of routine health checks in veterinary practice, since 3 months and 12 months are ages when cats are routinely seen by veterinarians (i.e. second vaccination and first annual booster vaccination, respectively). However, before such an approach is recommended, its ability to predict OW in adulthood should be tested in other cat populations, most notably in a larger outbred population of client-owned pet cats. This would enable the effects of various owner-related factors to be determined, especially feeding practices. Most notably, the present study did not consider the effects of feeding different types of main meal (e.g. dry food $v$. wet food) and giving additional foods such as table scraps or treats. Only once such studies have been conducted will the true value of early weight measurement be known.

The study findings must be considered in light of a number of limitations, most important of which was the fact that a colony of cats was used, rather than a population of pet cats. As such, the findings may not be fully reflective of all 
domesticated cats. Furthermore, the study population was small and, although both pedigree and mixed-breed cats were included, not all breeds were studied. There are also likely to be differences in the genetic background and degree of relatedness of these cats, compared with cats in different countries and on different continents. In addition to genetic factors, the use of colony cats means that the influence of the owner cannot be ascertained, and this may influence the generalisability of these results to the population as a whole. Environmental influences would also be less variable with all cats living in a similar environment, with similar climate exposure, lighting and opportunity for controlled exercise. As a result, the findings should be considered preliminary, and should be confirmed with epidemiological studies in pet cat populations and other colonies. Despite the limitations of a colony study, there are some notable advantages, most importantly that greater environmental control improves the ability to examine the physiological mechanisms in a smaller number of cats. The colony setting also enabled precise and accurate measurement of FI, through the use of electronic weighing scales. This ensured that FI was accurately measured, and known to be free from the inevitable imprecision that arises when owners estimate main meal intake, as well as the feeding of table scraps and treats. Although FI data were not available before cats were 3.5 years of age, and a variety of foods were fed over the timeframe of the study, all were maintenance diets, presented in a dry, extruded, kibbled format, and energy content was similar. Further, because every cat received the same two diets on each day, comparison between cats, and indeed between groups of cat, are still valid. Of course, there would be similar advantages inherent in the ability to weigh all cats, on a regular basis, using the same set of electronic weighing scales.

A further study limitation is that energy expenditure and physical activity were not measured, and the ambient temperature was not recorded. All these factors could have influenced FI and weight status. The similar environment would limit the influence of ambient temperature on the results; however, since not all cats had unlimited access to the outdoors, activity levels would probably be different among cats. That said, housing (i.e. exclusively indoor $v$. indoor-outdoor) was not found to be a significant influence on BW status although, arguably, our experimental design did not mimic the actual variability in housing conditions among the pet cat population. Further, an effect of exercise cannot be discounted altogether since there may have been individual differences in voluntary activity that were no accounted for. In future studies, methods of measuring physical activity should be considered, such as the use of accelerometers, that have now been validated for companion animals ${ }^{(42)}$.

Other limitations include the fact that group size was small for assessments made of weight change between 3 and 12 months of age. This might, arguably, have influenced the findings. Against this, however, the findings from these sixteen cats mirrored those of the larger forty-two-cat subgroup, and many of the findings were highly significant. A final issue was the fact that body composition was not assessed during the course of the study and, instead, we relied on
$\mathrm{BW}$ and BCS. In this respect, BCS was ideal in all cats at $\mathrm{BW} 1 \mathrm{y}$, suggesting that this technique may not be sensitive enough to detect changes significantly different between cats at this age. Further studies should now be considered to examine differences in body composition during growth in cats of OW and lean phenotype. This would enable the reason for the 12-month BW differences to be determined. For such studies, suitable research techniques would include dual-energy $\mathrm{X}$-ray absorptiometry ${ }^{(43)}$ or deuterium oxide ${ }^{(44)}$, although such methods would not be practical for large-scale studies in pet cat populations. Further, they are unlikely to be integrated into first-opinion veterinary practice in the near future and, for that reason, the relative change in BW between 3 and 12 months would be a more readily calculable and practical measure. As long as the same scales are used, the precision of such a measure is likely to be better than body composition methods. In this regard, while dual-energy X-ray absorptiometry is relatively precise for repeat measurements, its precision $^{(26)}$ is worse than for scales ${ }^{(45)}$.

In conclusion, $\mathrm{BW}$ at 1 year of age is a significant risk factor for colony cats fed ad libitum becoming OW by 8.5 years and this is, in turn, dictated by the rate of growth between 3 and 12 months of age. Further investigations are necessary for understanding which factors during growth (e.g. genetics or FI regulation) lead to this difference of $\mathrm{BW}$ at 1 year of age.

\section{Acknowledgments}

The authors thank all caregivers for participating in the study. This research received no specific grant from any funding agency in the public, commercial or not-for-profit sectors. Conflicts of interest are as follows: all the authors are employees of Royal Canin, except A. J. G., whose senior lectureship at the University of Liverpool is supported by Royal Canin. All the authors were involved in the study design, in the collection, analysis and interpretation of data, in the writing of the manuscript, and in the decision to submit the manuscript for publication. From the end of this study to the present, all the cats involved in the feeding performance studies have remained in ideal body condition.

\section{References}

1. Kopelman PG (2000) Obesity as a medical problem. Nature 404, 635-643.

2. Rennie KL \& Jebb SA (2005) Prevalence of obesity in Great Britain. Obesity Rev 6, 11-12.

3. Guo SS \& Chumlie WC (1999) Tracking of body mass index in children in relation to overweight in adulthood. Am J Clin Nutr 70, Suppl., 145S-148S.

4. Parsons TJ, Power C, Logan S, et al. (1999) Childhood predictors of adult obesity: a systematic review. Int J Obesity 23, Suppl. 8, S1-S107.

5. Kvaavick E, Tell GS \& Klepp K-I (2003) Predictors and tracking of body mass index from adolescence into adulthood. Arch Pediatr Adolesc Med 157, 1212-1218.

6. Kvaavik E, Klepp K-I, Tell GS, et al. (2009) Physical fitness and physical activity at age 13 years as predictors of cardiovascular disease risk factors at ages $15,25,33$, and 40 years: extended follow-up of the Oslo youth study. Pediatrics 123, e80-e86. 
7. Engeland A, Borge T, Tverdal A, et al. (2004) Obesity in adolescence and adulthood and the risk of adult mortality. Epidemiology $15,79-85$.

8. Reilly JJ, Armstrong J, Dorosty AR, et al. (2005) Early life risk factors for obesity in childhood: cohort study. BMJ 330, 1357-1359.

9. Ogden CL, Carroll MD, Curtin LR, et al. (2006) Prevalence of overweight and obesity in the United States, 1999-2004. J Am Med Assoc 295, 1549-1555.

10. Whitaker RC, Wright JA, Pepe MS, et al. (1997) Predicting obesity in adulthood from childhood and parental obesity. $N$ Engl J Med 337, 869-873.

11. Kruger R, Kruger HS \& MacIntyre UE (2006) The determinants of overweight and obesity among 10- to 15- year-old schoolchildren in the North West Province, South Africa - the THUSA BANA (Transition and Health during Urbanisation of South Africans; BANA, children) study. Public Health Nutr 9, 351-358.

12. Sjöberg A, Moraeus L, Yngve A, et al. (2011) Overweight and obesity in a representative sample of schoolchildren - exploring the urban-rural gradient in Sweden. Obes Rev 12, 305-314.

13. Baird J, Fisher D, Lucas P, et al. (2005) Being big or growing fast: systematic review of size and growth in infancy and later obesity. BMI 331, 929-931.

14. Scarlett JM \& Donoghue S (1998) Associations between body condition and disease in cats. J Am Vet Med Assoc 212, 1725-1731.

15. Lund EM, Armstrong PJ, Kirk CA, et al. (2005) Prevalence and risk factors for obesity in adult cats from private US veterinary practices. Int J Appl Res Vet Med 3, 88-96.

16. German AJ (2006) The growing problem of obesity in dogs and cats. J Nutr 136, 1940S-1946S.

17. Colliard L, Paragon B, Lemuet B, et al. (2009) Prevalence and risk factors of obesity in an urban population of healthy cats. J Feline Med Surg 11, 135-140.

18. Courcier EA, O’Higgins R, Mellor DJ, et al. (2010) Prevalence and risk factors for feline obesity in a first opinion practice in Glasgow, Scotland. J Feline Med Surg 12, 746-753.

19. Cave NJ, Allan FJ, Schokkenbroek SL, et al. (2012) A crosssectional study to compare changes in the prevalence and risk factors for feline obesity between 1993 and 2007 in New Zealand. Prev Vet Med 107, 121-133.

20. Sloth C (1992) Practical management of obesity in dogs and cats. J Small Anim Pract 33, 178-182.

21. Scarlett JM, Donoghue S, Saidla J, et al. (1994) Overweight cats prevalence and risk factors. Int J Obes 18 , S22-S28.

22. Robertson ID (1999) The influence of diet and other factors on owner-perceived obesity in privately owned cats from metropolitan Perth, Western Australia. Prev Vet Med 40, 75-85.

23. Russell K, Sabin R, Holt S, et al. (2000) Influence of feeding regimen on body condition in the cat. J Small Anim Pract 41, 12-17.

24. Allan FJ, Pfeiffer DU, Jones BR, et al. (2000) A cross-sectional study of risk factors for obesity in cats in New Zealand. Prev Vet Med 46, 183-196.

25. Laflamme DP (1997) Development and validation of a body condition score for cats: a clinical tool. Feline Pract 25, 13-18.

26. Association of American Feed Control Officials (AAFCO) (2003) Dogs and cats nutrient profiles. In AAFCO Official Publication, pp. 125-140. Oxford: AAFCO.

27. Chevallier S, Bertrand D, Kohler A, et al. (2006) Application of PLS-DA in multivariate image analysis. J Chemometrics 20, 221-229.
28. Druet C, Steller N, Sharp S, et al. (2012) Prediction of childhood obesity by infancy weight gain: an individual-level meta-analysis. Paediatr Perinat Epidemiol 26, 19-26.

29. Stettler N, Stallings VA, Troxel AB, et al. (2005) Weight gain in the first week of life and overweight in adulthood: a cohort study of European American subjects fed infant formula. Circulation 111, 1897-1903.

30. Maes HH, Neale MC \& Eaves LJ (1997) Genetic and environmental factors in relative body weight and human adiposity. Behav Genet 27, 325-351.

31. Atwood LD, Heard-Costa NL, Cupples LA, et al. (2002) Genomewide linkage analysis of body mass index across 28 years of the Framingham Heart Study. Am J Hum Genet 71, 1044-1050.

32. Haring T, Wichert B, Dolf G, et al. (2011) Segregation analysis of overweight body condition in an experimental cat population. J Hered 102, Suppl. 1, S28-S31.

33. Rhee KE, Phelan S \& McCaffery J (2012) Early determinants of obesity: genetic, epigenetic, and in utero influences. Int J Pediatr 2012, article ID 463850.

34. Oken E, Rifas-Shiman SL, Field AE, et al. (2008) Maternal gestational weight gain and offspring weight in adolescence. Obstetr Gynecol 112, 999-1006.

35. Godfrey KM, Sheppard A, Gluckman PD, et al. (2011) Epigenetic gene promoter methylation at birth is associated with child's later adiposity. Diabetes 60, 1528-1534.

36. Gillman MW, Rifas-Shiman S, Berkey CS, et al. (2003) Maternal gestational diabetes, birth weight, and adolescent obesity. Pediatrics 111, 221-226.

37. Al Mamun DA, Lawlor R, Alati MJ, et al. (2006) Does maternal smoking during pregnancy have a direct effect on future offspring obesity? Evidence from a prospective birth cohort study. Am J Epidemiol 164, 317-325.

38. Vester BM, Belisto KR \& Swanson KS (2012) Serum metabolites, ghrelin and leptin are modified by age and/or diet in weanling kittens fed either a high- or moderate-protein diet. Am Sci J 83, 426-433.

39. German AJ, Holden SL, Mather NJ, et al. (2011) Low maintenance energy requirements of obese dogs after weight loss. Br J Nutr 106, S93-S96.

40. German AJ (2011) Editorial: obesity - weighing on the mind of the owner? I Small Anim Pract 52, 619-620.

41. Ekelund U, Ong K, Linné Y, et al. (2006) Upward weight percentile crossing in infancy and early childhood independently predicts fat mass in young adults: the Stockholm Weight Development Study (SWEDES). Am J Clin Nutr 83, 324-330.

42. Wrigglesworth DJ, Mort ES, Upton SL, et al. (2011) Accuracy of the use of triaxial accelerometry for measuring daily activity as a predictor of daily maintenance energy requirement in healthy adult Labrador retrievers. Am J Vet Res 72, 1151-1155.

43. Raffan E, Holden SL, Cullingham F, et al. (2006) Standardised positioning is essential for precise determination of body composition using dual-energy X-ray absorptiometry. J Nutr 136, 1976S1978S.

44. Burkholder WJ \& Thatcher CD (1998) Validation of predictive equations for use of deuterium oxide dilution to determine body composition of dogs. Am J Vet Res 59, 927-937.

45. Burkholder WJ (2001) Precision and practicality of methods assessing body composition of dogs and cats. Comp Cont Edu Pract Vet 23, $1-10$. 